Revista Mexicana de Economía y Finanzas Nueva Época

Volumen 14 Número 2, Abril-Junio 2019, pp. 261-277

DOI: https://doi.org/10.21919/remef.v14i2.299

\title{
Diagnóstico de la complejidad económica del estado de Hidalgo: de las capacidades a las oportunidades
}

\author{
Carla Carolina Pérez Hernández ${ }^{1}$
}

Universidad Autónoma del Estado de Hidalgo, México

Blanca Cecilia Salazar Hernández

Universidad Autónoma del Estado de Hidalgo, México

Jessica Mendoza Moheno

Universidad Autónoma del Estado de Hidalgo, México

(Recepción: 8/junio/2018, aceptado: 29/noviembre/2018)

\section{Resumen}

El objetivo del presente trabajo es examinar las oportunidades de diversificación productiva y de emprendimiento que derivan de las capacidades existentes y potenciales con las que cuenta específicamente el estado de Hidalgo. Para ello, se desarrolló un estudio empírico haciendo uso del Método de Reflexiones (MR) y la asignación de ponderadores, con base en las variables de la Teoría de Complejidad Económica propuestas por Hidalgo Hausmann (2009) y recopiladas del Atlas de Complejidad (2018). A partir de las operaciones algebraicas, se halla en un primer momento que el estado de Hidalgo se encuentra actualmente ubicado en el cuadrante que refleja: "mayor diversificación promedio con mayor ubicuidad promedio". En un segundo momento, la estrategia balanceada de ponderación, logra generar un ranking de oportunidades que puede ser útil para argumentar modificaciones en materia de política industrial y también para orientar y validar proyectos productivos de emprendedores, empresarios e inversionistas en la región. Una limitación del trabajo es que se basa sólo en datos provenientes de la economía formalindustrial y su originalidad radica en que no existen estudios previos en el contexto analizado.

Clasificación JEL: O10, O30, O31

Palabras clave: complejidad económica, capacidades y oportunidades, ubicuidad, diversidad

\section{Diagnosis of the Economic Complexity in the State of Hidalgo: From Capabilities to Opportunities}

\section{Abstract}

The objective of this work is to examine the opportunities for productive diversification and entrepreneurship that derive from the existing and potential capacities of the state of Hidalgo. To this end, an empirical study was developed using the Reflections Method (RM) and the assignment of weights, based on the variables of the Economic Complexity Theory proposed by Hidalgo Hausmann (2009) and compiled from the Atlas of Economic Complexity (2018). From the algebraic operations, it is at first sight that the state of Hidalgo is currently located in the quadrant that reflects: "greater average diversification with greater average ubiquity". Secondly, the balanced weighting strategy manages to generate a ranking of opportunities that may be useful to argue modifications in industrial policy and also to guide and validate productive projects of entrepreneurs, businessmen, and investors in the region. A limitation of the work is that it is based only on data from the formal industrial economy; its originality lies in the fact that there are no previous

\footnotetext{
${ }^{1}$ Circuito La Concepción km. 2.5 Col. San Juan Tilcuautla, San Agustín Tlaxiaca; Hidalgo, México. C.P 42160. Tel: +52 7711355545 Correo: carolina.cph@gmail.com *Fuente de financiamiento: PRODEP
} 
REMEF (The Mexican Journal of Economics and Finance)

\section{Abstract}

studies in the context analyzed.

JEL Classification: O10, O30, O31

Keywords: Economic complexity, Capacities and opportunities, Ubiquity, Diversity

\section{Introducción}

Las capacidades son insumos no transables. La diferencia en productividad e ingresos entre entidades puede ser explicada por diferencias en la complejidad económica, entendida como la diversidad de las capacidades presentes en cada entidad (Hausmann, Cheston Santos, 2015).

Felipe, Kumar Abdon (2010), plasman la premisa que "sembrar mejores capacidades, lleva a cosechar mejores oportunidades", las capacidades entonces engendran oportunidades, por lo que primordialmente es necesario analizar las capacidades existentes para detectar el abanico de oportunidades que una entidad puede tener. Por lo tanto, la presente investigación surge con la necesidad de reconocer las oportunidades de diversificación productiva y/o de emprendimiento que derivan de las capacidades existentes y potenciales con las que cuenta específicamente el estado de Hidalgo. Se establece entonces la siguiente pregunta central: ¿Cuáles son las oportunidades concretas de diversificación productiva factibles en el corto, mediano y largo plazo identificadas a partir de las capacidades productivas existentes a nivel local? Claramente el resultado de investigación será un trio de rankings de oportunidades de emprendimiento y diversificación productiva que pueden ser útiles para diversos agentes.

Por ende, dar respuesta a la interrogante central de investigación, resulta ampliamente relevante para el sector gubernamental, ya que los hallazgos derivados del estudio pueden argumentar modificaciones o ajustes en materia de política industrial focalizada que promuevan sectores con potencial emergente. Con los resultados arrojados, es posible validar o corregir las agendas de innovación (en este caso la del estado de Hidalgo). Además, la información resultante podrá ser útil para los emprendedores, empresarios, inversionistas y diversos agentes del ecosistema emprendedor, ya que podrían utilizar los rankings de oportunidades para validar la puesta en marcha de sus proyectos productivos, corroborando la preexistencia de capacidades adyacentes que los puedan impulsar. La hipótesis fundamental es que "los nuevos productos que desarrolla una entidad dependen sustancialmente de las capacidades ya disponibles en esa entidad" (Hidalgo Hausmann, 2009).

Es ampliamente reconocido que las entidades tienden a diversificar sus exportaciones al ingresar productos que están relacionados con su exportaciones actuales (Pinheiro et al, 2018). Y es por ello que según Abdon Felipe (2010), se hace necesaria la acumulación de nuevas capacidades para mejorar el nivel de producción y generar nuevas oportunidades hacia una transformación estructural (que pasa de la producción de actividades menos sofisticadas a actividades más sofisticadas). No obstante, primeramente es necesario que las entidades reconozcan cuáles son sus capacidades actuales y proyecten con base en ello, las oportunidades productivas a las cuales pueden incursionar de forma competitiva y factible. La hipótesis del estudio remarca la existencia de un abanico de oportunidades para que el estado de Hidalgo se mueva de manera gradual hacia productos de mayor diversificación y sofisticación.

La organización del trabajo se detalla de la siguiente manera: en una primera sección se muestra en breve la revisión de la literatura, posteriormente se desglosa la metodología utilizada, para continuar con el análisis de resultados e interpretaciones y finalmente se emiten las conclusiones y reflexiones del estudio. 


\section{Revisión de la literatura}

La teoría de Legos de la Complejidad Económica, es una corriente reciente del pensamiento económico, fue desarrollada por Hidalgo Hausmann (2009), quienes argumentan que es posible crear medidas indirectas de las capacidades disponibles en un país o entidad pensando que cada capacidad puede ser vista como una pieza de Lego (bloque). En esta analogía, un producto es equivalente a un modelo de Lego, y un país es equivalente a una cubeta de Legos. Los países o entidades podrán hacer ciertos productos si cuentan con todas las capacidades necesarias, al igual que un niño es capaz de producir un modelo de Lego siempre y cuando el balde de Legos contenga todas las piezas necesarias. Por consecuencia, la complejidad económica es equivalente a preguntar si podemos inferir propiedades como la diversidad y la exclusividad de las piezas de Legos (capacidades) mirando sólo los modelos construidos por un grupo de niños. La respuesta es sí y por ende la diversidad y ubicuidad en los territorios son parámetros esenciales en la teoría de la complejidad económica que deben ser medidos y contrastados.

La teoría de la complejidad económica ha tenido gran difusión internacional principalmente gracias al observatorio de complejidad económica, creado en el MIT. El cual sirve como herramienta virtual y analítica para entender las dinámicas del desarrollo económico (Simoes Hidalgo, 2011).

Adicionalmente, un hecho bien establecido en la literatura sobre economía internacional y regional es que los países y entidades tienen más probabilidades de ingresar a actividades económicas que son similares a las que ya tienen. Este principio de relación se ha observado para actividades tan diversas como los productos, las industrias, tecnologías y áreas de investigación (Pinheiro et al, 2018). Derivado de lo anterior, numerosos estudios empíricos a nivel país han sido desarrollados en aras de proveer orientación sobre oportunidades de diversificación y necesidades de acumulación de capacidades (Felipe, Kumar, Abdon, Bacate, 2012; Hausmann, Hidalgo, Bustos, et al., 2014; Hausmann Hidalgo, 2013; Nepelski De Prato, 2015).

\subsection{Capacidades y Oportunidades}

Al final lo que un país produce (hace) es reflejo de sus capacidades y conocimientos (Hidalgo, 2017) y es ampliamente reconocido que los individuos o sociedades más desarrollados no dependen tanto de sus recursos, como de lo que son "capaces de hacer" con los recursos que tienen (Ackoff, 2000). La capacidad de hacer supera a la capacidad de tener. Por ende, en los lugares donde florece la innovación (territorios innovadores) por lo general glorifican más las capacidades que el dinero (Oppenheimer, 2014). ¿Cuál es la diferencia entonces entre los humanos y el resto de las especies? La respuesta es que los humanos somos capaces de crear representaciones físicas de los objetos que imaginamos, mientras que las demás especies deben limitarse al inventario que ofrece la naturaleza. Tenemos pues la capacidad de hacer lo que imaginamos. En ese sentido, el desarrollo económico no se basa en la capacidad de consumir de una parte de la economía, sino en la capacidad que tienen las personas de hacer sus sueños realidad: el desarrollo económico no es la capacidad de comprar, sino la capacidad de hacer (Hidalgo, 2017). Por tanto, la calidad de vida, no está determinada por las "utilidades" sino que por las "capacidades", es decir, las características de las personas que les permiten "funcionar" en el mundo y acceder a una vida más plena. (Sen; citado por Cepal, 2005). Por otra parte, es ampliamente reconocido que el descubrimiento de oportunidades constituye una habilidad emprendedora importante, una fuente de ventaja competitiva y un componente que debería ser importante en la formación proporcionada a individuos en materia de creación de empresas (González Solís, 2011).

En ese sentido, la literatura sobre . ${ }^{\circ}$ portunidades empresariales"ha crecido rápidamente. Al dirigir la atención a las primeras etapas de desarrollo de nuevas actividades 
REMEF (The Mexican Journal of Economics and Finance)

Diagnóstico de la complejidad económica del estado de Hidalgo:

económicas y organizaciones, marcando una reorientación acertada de la investigación empresarial (Davidsson, 2015). Se destaca entonces que antes de que el cambio tecnológico lleve a nuevos procesos, productos, mercados o formas de organización, los empresarios deben descubrir oportunidades para explotar la nueva tecnología (Shane, 2000). Se reconoce entonces que "la actividad emprendedora está impulsada por la percepción de oportunidades combinadas con las habilidades y las motivaciones para explotarlas. La oportunidad se refiere tanto a la existencia, como a la percepción de oportunidades disponibles" (GEM, 2005, p.12).

En México, es posible identificar incipientes estudios empíricos que analizan las "oportunidades" de diversificación productiva a través de las "capacidades". Como el análisis de Hausmann, Cheston Santos (2015), quienes clasifican en tres, las estrategias para la identificación de oportunidades con base en capacidades: 1) La Estrategia Balanceada: que consiste en identificar los adyacentes posibles, y los conjuntos de productos más atractivos considerando los indicadores de distancia a la capacidad, complejidad, y valor de oportunidad. 2) La Estrategia de Apuestas: las cuales son estrategias alternativas que priorizan mayor el valor de oportunidad futuro y 3) La Estrategia de Fruta Madura: la cual identifica oportunidades con base en los productos más adyacentes o de menor distancia a la capacidad actual.

Recientemente Castañeda (2018), desarrolló una serie de reportes sub-nacionales de la complejidad económica para 16 entidades mexicanas. Sin embargo, no se encuentran estudios previos enfocados al contexto hidalguense que apliquen los parámetros de complejidad, diversidad y ubicuidad que sean ocupados para identificar oportunidades de diversificación y emprendimiento productivo propios de dicha región.

\section{Metodología y Datos}

La presente investigación ocupa los datos del Atlas de la Complejidad Económica (2018). La metodología consiste en desarrollar un estudio empírico en dos vías, la primera radica en aplicar el Método de Reflexiones (MR) para mapear la ubicuidad y diversidad industrial de las entidades mexicanas para el año 2014 (lapso disponible más reciente) con base en la matriz binaria, $M_{s, a}$ que indica las actividades económicas en las que se especializa cada entidad, utilizando el Índice de Ventaja Comparativa Revelada $V C R$, desglosando entonces las ecuaciones (3), (4) y (5), con el objetivo de identificar la posición que ocupa el estado de Hidalgo a nivel nacional en materia de ubicuidad-diversidad industrial, posteriormente, la segunda vertiente consiste en desglosar específicamente para el estado de Hidalgo, las estrategias de diversificación productiva que reflejan las oportunidades de corto, mediano y largo plazo viables de optar; con base en cuatro indicadores: Densidad $\left(x_{1}\right)$, Índice de Complejidad del Producto $\left(x_{2}\right)$, Índice de Complejidad Potencial del Producto $\left(x_{3}\right)$, y valor nacional de las exportaciones por industria $\left(x_{4}\right)$. Dichos indicadores tendrán el siguiente tratamiento: A) Estimación de la media de la variable: complejidad del producto $\left(x_{2}\right)$, para discriminar y eliminar del análisis aquellos productos que ofrecen una complejidad inferior al promedio de la entidad. B) Normalización de los indicadores bajo la formula $\frac{x_{n}-\overline{x_{n}}}{\sigma}$ para los tres primeros indicadores, mientras que para el valor nacional de las exportaciones por industria $\left(x_{4}\right)$ se normaliza por logaritmo natural (Ln), C) Aplicación de ecuación (7) para las tres diferentes estrategias de diversificación y el hallazgo de oportunidades de emprendimiento y finalmente D) Interpretación de los resultados obtenidos. Las fases mencionadas se desglosarán analíticamente en las siguientes secciones. 


\subsection{La teoría de legos de la complejidad económica (proceso me- todológico)}

A pesar que "las capacidades no son claramente conmensurables puesto que son irreductiblemente diversas" (Sen, 2015, p.270) hoy en día, es posible cuantificar las capacidades productivas de las economías haciendo uso de la teoría de la complejidad económica, la cual hace mediciones sobre la sofisticación de la estructura productiva de una entidad, es decir la diversidad contada por el número de productos que exporta un lugar y la ubicuidad de esos productos, entendida como el número de entidades que exportan el mismo producto (Hidalgo Hausmann, 2009). Claramente, las economías más sofisticadas tienen un alto grado de diversidad y exportan productos que en promedio tienen una baja ubicuidad, lo que implica que sólo unas pocas entidades diversificadas pueden elaborar dichos productos sofisticados.

En ese sentido, la Ventaja Comparativa Revelada (VCR), es un indicador clave de la complejidad económica, el cual refleja si una entidad posee ventajas comparativas en la elaboración de un bien. Entonces, la VCR de una entidad $(s)$ en la actividad productiva $(\alpha)$ esta denotada de la siguiente manera:

$$
V C R_{s, \alpha}=\frac{\frac{X_{s, \alpha}}{\sum_{\alpha^{\prime}}^{n} X_{s, \alpha^{\prime}}}}{\sum_{s^{\prime} \alpha}^{s_{s^{\prime}}, \alpha}}
$$

Donde $X_{s . \alpha}$ es el total de exportaciones de la entidad $s$, en la actividad $\alpha$, por su parte, $\sum_{\alpha^{\prime}}^{n} X_{s, \alpha^{\prime}}$ son las exportaciones totales en la entidad $s$. Mientras que $\sum_{s^{\prime} \alpha}^{n} X_{s^{\prime}, \alpha}$, representa las exportaciones totales en el sector en todo el país; análogamente, $\sum_{s^{\prime} \alpha^{\prime}}^{n} X_{s^{\prime}, \alpha^{\prime}}$ es el total de exportaciones en todo el país. Si esta relación es mayor que 1, se dice que el lugar tiene ventaja comparativa revelada en dicho sector o producto. La VCR es también usada para definir la matriz binaria ${ }^{2} M_{s, \alpha}$ la cual es igual a 1 si el lugar s tiene una $V C R \geq 1$ y cero en otros casos. Esto es:

$$
M_{s, \alpha}=1 \text { si } V C R_{s, \alpha} \geq 1
$$

De acuerdo con Hidalgo Hausmann (2009), la matriz $M_{s, \alpha}$ permite definir el nivel de ubicuidad y diversidad de los productos y puede representarse también a escala subnacional como $M_{s, a}$. Donde los estados están representados por $(s)$, mientras que las actividades económicas quedan denotadas por $(\alpha)$. Algebraicamente:

$$
\begin{aligned}
& \text { Diversidad }=k_{s, 0}=\sum_{\alpha=1}^{n} m_{s, a} \\
& \text { Ubiquidad }=k_{\alpha, 0}=\sum_{s=1}^{32} m_{s, a}
\end{aligned}
$$

Posteriormente, tal como sugieren Hidalgo Hausmann, (2009); Felipe et al. (2012); Hartmann et al (2017), haciendo uso del Método de Reflexiones (MR) se puede definir una matriz que conecte a las entidades que exportan productos similares, ponderándolas por el inverso de la ubicuidad de un producto (para descontar productos comunes), y normalizando por la diversidad de las entidades. Desde un punto de vista matemático, el MR se define como:

$$
k_{s, N}=\frac{1}{k_{s, 0}} \sum_{a} M_{s a^{k}} a, N-1
$$

\footnotetext{
${ }^{2}$ Hidalgo Hausmann (2009), proponen usar $V C R \geq 1$ como umbral; esto implica que el país o entidad, está especializada en la actividad económica o producto en cuestión.
} 
REMEF (The Mexican Journal of Economics and Finance) Diagnóstico de la complejidad económica del estado de Hidalgo:

$$
k_{\alpha, N}=\frac{1}{k_{\alpha, 0}} \sum_{s} M_{s a^{k}} s, N-1
$$

Las ecuaciones (5) y (6), recalcan que MR consiste en calcular iterativamente el valor promedio de las propiedades del nivel previo de un nodo vecino y se define como el conjunto de observables, en dónde $N \geq 1$. Además, $k_{s, 0}, k_{\alpha, 0}$ representan repectivamente, los niveles observados de diversificación de una entidad y de la ubicuidad de una actividad productiva. Por lo tanto, se caracteriza cada entidad por medio del vector $\vec{k}_{s}=\left(k_{s, 0}, k_{s, 1}, k_{s, 2} \ldots k_{s, N}\right)$ y cada actividad económica con el vector $\vec{k}_{\alpha}=\left(k_{\alpha, 0}, k_{\alpha, 1}, k_{\alpha, 2} \ldots k_{\alpha, N}\right)$. En donde, de acuerdo con Hidalgo Hausmann (2009) el diagrama de ubiquidad y diversidad se construye con las fórmulas (3), (4) y (5), utilizando entonces los vectores $k_{s, 0}-k_{s, 1}$, que expresan como resultado los 4 cuadrantes del nivel de diversidad y ubiquidad promedio.

Hasta este punto, la metodología ubica a los 32 estados de la república mexicana de acuerdo con su respectivo nivel de diversidad y ubiquidad. Resaltando entonces el cuadrante de pertenencia del estado de Hidalgo. No obstante para completar el estudio focalizado específicamente al estado de Hidalgo, es necesario realizar el siguiente tratamiento de datos.

Primeramente, para definir los rankings de oportunidades identificados por medio de las capacidades, fue necesario recolectar los indicadores de complejidad económica relacionados con: Densidad $\left(x_{1}\right)$, Índice de Complejidad del Producto $\left(x_{2}\right)$, Índice de Complejidad Potencial del Producto $\left(x_{3}\right)$, y Valor nacional de las exportaciones por industria $\left(x_{4}\right)$. Dichas variables reflejan las características de las capacidades productivas actuales y futuras. Tal como se observa en las definiciones tabla 1.

Tabla 1. Datos de complejidad económica

\begin{tabular}{|l|l|}
\hline Nombre de la variable & \multicolumn{1}{|c|}{ Definición } \\
\hline Densidad $\left(x_{1}\right)$ & $\begin{array}{l}\text { La densidad varía entre 0 y 1, los valores más grandes indican que } \\
\text { la entidad ha avanzado una ventaja comparativa en muchos bienes } \\
\text { próximos al bien i y tiene mayor probabilidad de exportarlo en el } \\
\text { futuro. }\end{array}$ \\
\hline $\begin{array}{l}\text { Índice de } \\
\text { Complejidad del } \\
\text { Producto }\left(x_{2}\right)\end{array}$ & $\begin{array}{l}\text { Ordena los productos de exportación según la diversidad y } \\
\text { ubicuidad de capacidades productivas requeridas para su } \\
\text { fabricación. Un lugar con alta complejidad económica es capaz de } \\
\text { producir muchos bienes y servicios que en promedio pocos saben } \\
\text { hacer. }\end{array}$ \\
\hline $\begin{array}{l}\text { Indice de } \\
\text { Complejidad } \\
\text { Potencial del }\end{array}$ & $\begin{array}{l}\text { Mide en qué medida se podría beneficiar un lugar si consigue } \\
\text { desarrollar una industria o producto de exportación específico. } \\
\text { También conocida como "ganancia de oportunidad", esta medida } \\
\text { representa la distancia a todos los otros sectores (o exportaciones) } \\
\text { que un lugar no produce actualmente y su respectiva complejidad. } \\
\text { Refleja cómo un nuevo sector (o exportación) puede abrir paso a } \\
\text { otros sectores o productos más complejos. }\end{array}$ \\
\hline $\begin{array}{l}\text { Valor nacional de las } \\
\text { exportaciones por } \\
\text { industria }\left(x_{4}\right)\end{array}$ & $\begin{array}{l}\text { Monto total de las exportaciones por sector industrial expresados } \\
\text { en dólares. }\end{array}$ \\
\hline
\end{tabular}

Fuente: Elaboración propia con base en Atlas de Complejidad (2018).

Una vez normalizadas ${ }^{3}$ todas variables y siguiendo a Castañeda (2018), se determinan las tres estrategias de selección de oportunidades:

1) La Estrategia al alcance o de Fruta Madura (para el corto plazo): identifica oportunidades cuando se trata de productos que requieren de capacidades relativa-

\footnotetext{
${ }^{3}$ Normalización de los indicadores bajo la formula $\frac{x_{n}-\overline{x_{n}}}{\sigma}$ para los tres primeros indicadores, mientras que para el valor nacional de las exportaciones por industria $\left(x_{4}\right)$ se normaliza por logaritmo natural (Ln).
} 
mente similares a las existentes en la localidad pero que no se exportan o aún no son competitivos exportadores en la región.

2) La Estrategia Balanceada (para el mediano plazo): identifica oportunidades cuando se le da un peso similar a la sofisticación del producto y a su conectividad con otros productos atractivos.

3) La Estrategia de Apuestas a futuro (para el largo plazo): otorga mayor importancia a las oportunidades que abre una industria en el espacio de productos. En otras palabras, se asigna mayor peso a la "ganancia de oportunidad. en relación con su probabilidad de exportarlo en el futuro.

Habiendo definido las estrategias, se procede a ponderarlas, es decir asignarles un peso (w) en función de la importancia que cada indicador en particular tiene dentro de cada estrategia. Tal como se observa a continuación:

$$
\text { Estrategias de seleccin }=\sum_{i=1}^{n} X_{i} W_{i}=x_{1} w_{1}+x_{2} w_{2}+x_{3} w_{3}+x_{4} w_{4}
$$

Donde:

\begin{tabular}{|c|c|c|}
\hline \multicolumn{3}{|c|}{ Estrategias de selección (ponderaciones w) } \\
\hline Estrategia al alcance & Estrategia balanceada & Apuesta estratégica \\
\hline$w_{1}=0.7$ & $w_{1}=0.5$ & $w_{1}=0.4$ \\
$w_{2}=0.1$ & $w_{2}=0.2$ & $w_{2}=0.1$ \\
$w_{3}=0.1$ & $w_{3}=0.2$ & $w_{3}=0.4$ \\
$w_{4}=0.1$ & $w_{4}=0.1$ & $w_{4}=0.1$ \\
\hline Densidad $\left(x_{1}\right)$, Índice de Complejidad del Producto $\left(x_{2}\right)$, Índice de Complejidad Potencial \\
del Producto $\left(x_{3}\right)$, y Valor nacional de las exportaciones por industria $\left(x_{4}\right)$.
\end{tabular}

Nota: Todas las variables se encuentran previamente normalizadas.

Finalmente, se procede a realizar la interpretación de los resultados obtenidos en cada una de las fases metodológicas.

\section{Análisis de los resultados}

El análisis de los resultados se encausa en dos direcciones: primero, resaltar los hallazgos derivados del método de reflexiones (MR) que nos permitió mapear la ubicación del estado de Hidalgo en materia de ubicuidad y diversidad (aplicando las ecuaciones 1-5). Y en un segundo momento, revelar los rankings de oportunidades de emprendimiento y diversificación productiva derivados de la ecuación (7).

\subsection{Análisis del Método de Reflexiones (MR).}

Inicialmente, se genera la matriz compleja de orden $32 \times 307$, donde las filas (32) están conformadas por las entidades mexicanas $\left(s_{n}\right)$ y las columnas (307) indican las actividades productivas $\left(\alpha_{n}\right)$ comprendidas por todos los sectores industriales que exportan (utilizando la versión mexicana del Sistema de Clasificación Industrial de América del Norte -SCIAN-, revisión de 2007). Los valores que integran la matriz reflejan los resultados de la $\mathrm{VCR}^{4}$ de cada actividad para cada estado.

\begin{tabular}{c|cccccccc} 
& $\alpha_{1}$ & $\alpha_{2}$ & $\alpha_{3}$ & $\alpha_{4}$ & $\alpha_{5}$ & $\ldots$ & $\alpha_{307}$ & \\
Aguascalientes & 0.54 & 0.93 & 0.54 & 1.28 & 0.30 & $\ldots$ & 0 & \\
Baja California & 0.28 & 6.08 & 1.58 & 0.88 & 1.55 & $\ldots$ & 0.42 \\
Hidalgo & 0.29 & 0.13 & 0.07 & 0.13 & 0.06 & $\ldots$ & 0 & \\
$\vdots$ & $\vdots$ & $\vdots$ & $\vdots$ & $\vdots$ & $\vdots$ & $\ddots$ & $\vdots$ \\
Zacatecas & 1.28 & 1.62 & 0.52 & 0.62 & 0.80 & $\ldots$ & 0 & \\
& & & & & & & &
\end{tabular} $32 \times 307$

\footnotetext{
${ }^{4}$ Los datos de la VCR son compilados del Atlas del complejidad económica (2018).
} 
REMEF (The Mexican Journal of Economics and Finance) Diagnóstico de la complejidad económica del estado de Hidalgo:

Se construye entonces la matriz binaria $M_{s a}$ usando la regla de decisión denotada en la ecuación (2). Dicha matriz muestra las actividades productivas en las que las entidades se especializan.

$$
M_{s a}=\left[\begin{array}{ccccccc}
0 & 0 & 0 & 1 & 0 & \cdots & 0 \\
0 & 1 & 1 & 0 & 1 & \cdots & 0 \\
0 & 0 & 0 & 0 & 0 & \cdots & 0 \\
\vdots & \vdots & \vdots & \vdots & \vdots & \ddots & \vdots \\
1 & 1 & 0 & 0 & 0 & \cdots & 0
\end{array}\right]
$$

$32 \times 307$

Posteriormente, se aplican las ecuaciones (3) y (4) que calculan la diversidad y ubicuidad de la matriz $M_{s a}$ respectivamente. Claramente, la diversidad resulta de sumar las filas de la matriz, mientras que la ubicuidad es resultado de la sumatoria de las columnas de la misma.

$$
\text { Diversidad }=k_{s, 0}=\left[\begin{array}{c}
87 \\
86 \\
109 \\
\vdots \\
75
\end{array}\right] \quad \text { Ubiquidad }=k_{\alpha, 0}=\left[\begin{array}{lllllll}
8 & 8 & 8 & 12 & 13 & \cdots & 5
\end{array}\right]
$$

$32 \times 1 \quad 1 \times 307$

Derivado de lo anterior, es posible observar que el estado de Hidalgo es el más diverso (industrialmente) al menos en comparación con Aguascalientes, Baja California y Zacatecas (entidades visibles en la notación algebraica). No obstante, una cosa es tener una producción relativamente más diversificada y otra muy distinta es lograr elaborar productos que pocas entidades sean capaces de hacer, es decir, tener menor ubicuidad y mayor sofisticación productiva que denote el arraigo de capacidades productivas, tecnológicas y organizativas más genuinas.

Por ende, es necesario ahora aplicar la ecuación (5) para identificar la ubicuidad promedio de las entidades mexicanas, siendo ésta normalizada por la diversidad calculada previamente. Tal como se observa a continuación:

$$
\begin{array}{r}
k_{s, 1}=\left[\begin{array}{ccccccc}
0 & 0 & 0 & 1 & 0 & \cdots & 0 \\
0 & 1 & 1 & 0 & 1 & \cdots & 0 \\
0 & 0 & 0 & 0 & 0 & \cdots & 0 \\
\vdots & \vdots & \vdots & \vdots & \vdots & \ddots & \vdots \\
1 & 1 & 0 & 0 & 0 & \cdots & 0
\end{array}\right] *\left[\begin{array}{c}
8 \\
8 \\
8 \\
\vdots \\
5
\end{array}\right]=\left[\begin{array}{c}
1056 / 87 \\
827 / 83 \\
1363 / 109 \\
\vdots \\
1059 / 75
\end{array}\right]=\left[\begin{array}{c}
12,13 \\
9,96 \\
12,50 \\
\vdots \\
14,12
\end{array}\right] \\
32 \times 307 \times 1
\end{array}
$$

Nota: La matriz de ubicuidad de orden $1 \times 307$, se transpone al orden $307 \times 1^{*}$.

Los hallazgos de la ecuación (5) muestran que aunque el estado de Hidalgo tiene cierto grado de diversidad (con 109 sectores de especialidad exportadora de 307 totales a nivel nacional), éste resultado se ve opacado por un alto grado de ubicuidad promedio (12.50, lo que implica que casi la mitad de los estados se especializan en los mismos sectores que Hidalgo), lo que refleja que dicha entidad elabora productos que la mayoría de las entidades mexicanas son capaces de exportar. No hay que olvidar, que las entidades que acervan mayores y mejores capacidades son aquellas que tienen una mezcla entre una elevada diversidad y una baja ubicuidad. Hidalgo necesita entonces disminuir su ubicuidad y para ello, la innovación tecnológica subyacente de capacidades tecnológicas y organizativas (formación, promoción y consolidación de habilidades y conocimientos en relación con mejores rutinas organizacionales) se torna crucial. Por el contrario, por 
ejemplo, el estado de Baja California tiene una diversidad baja (86) en combinación con una ubicuidad baja (9.96), por lo que su tarea primordial consiste en aumentar su diversidad. El caso de Baja California es relativamente más sencillo de resolver que el caso del Estado de Hidalgo. Porque resulta más fácil diversificar la productividad saltando a sectores cercanos, ostentando ya capacidades sofisticadas, que disminuir la ubicuidad mediante la sofisticación de productos y capacidades que aún no se ostentan.

Los resultados en extenso de las operaciones algebraicas desarrolladas previamente, pueden observarse en la Figura 1, la cual muestra que las entidades mejor posicionadas se agrupan en el cuarto cuadrante (alta diversificación y baja ubicuidad), estas entidades son: Jalisco, Estado de México, Nuevo León, Ciudad de México y Querétaro. Mientras que las entidades peor posicionadas son aquellas encasilladas en el primer cuadrante (baja diversidad y alta ubicuidad). Paralelamente, se observa que el Estado de Hidalgo se ubica en el segundo cuadrante, el cual manifiesta una diversidad mayor al promedio, pero una ubicuidad mayor al promedio. Finalmente, en el tercer cuadrante se conglomeran las entidades que reflejan una baja ubicuidad promedio (aspecto positivo) con una baja diversidad promedio (aspecto negativo).

Figura 1. Ubicuidad y diversidad industrial promedio de los estados: Año 2014

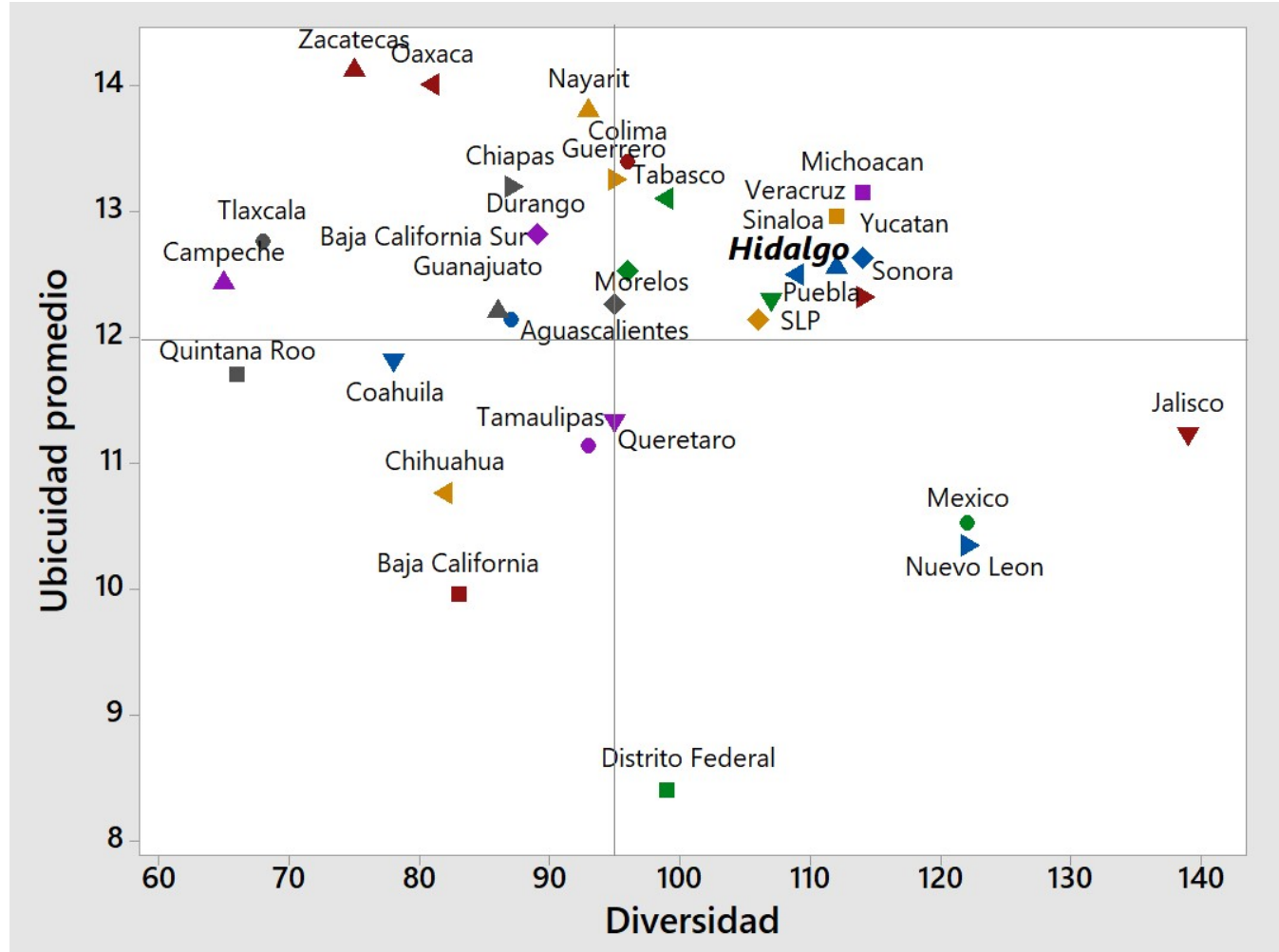

Nota: Dentro de los cuadrantes, el eje de las abscisas parte la ubicuidad nacional promedio, mientras que el eje de las ordenadas parte de la diversidad nacional promedio.

Fuente: Elaboración propia con base en cálculos de ubicuidad y diversidad (Minitab 17).

\subsection{Resultados de las estrategias de diversificación productiva: Rankings de oportunidades}

En las tablas 2, 3 y 4, se muestran los rankings de oportunidades con respecto a cada estrategia de selección, resultado de la ecuación (6). En este caso 3 productos aparecen en los 3 rankings: "Las demás manufacturas de hierro o acero", "remolques y semirremolques", "vidrieras aislantes de paredes múltiples". Lo cual implica que para estos productos 
REMEF (The Mexican Journal of Economics and Finance) Diagnóstico de la complejidad económica del estado de Hidalgo:

es más factible es que exista un mayor consenso sobre sus virtudes por parte de los actores, públicos y privados, involucrados en el diseño de políticas. Por otro lado, a partir de 2 criterios diferentes se repiten 16 productos: "Estufas y aparatos no eléctricos similares de fundición", "hierro o acero", "construcciones y sus partes de fundición", "artículos para la construcción, de plástico"; "las demás placas de plástico", "asientos", "los demás muebles y sus partes", "refrigeradores y congeladores", "bombas, compresores, ventiladores, etc"; "artículos de grifería para tuberías, calderas, etc"; "partes para motores de encendido por chispa", "arboles de transmisión", "partes de vehículos automóviles y tractores", "señales eléctricas para vías", "los demás impresos", "artículos textiles para usos técnicos". Lo que implica que para estos productos también existe un amplio grado de convergencia sobre la factibilidad de su elaboración.

La Estrategia al alcance o de Fruta Madura (para el corto plazo): identificó oportunidades provenientes de sectores maduros como: "Textiles y muebles", "metales" y "vegetales, alimentos y madera". No obstante, apareció el sector "químico y de plásticos". Bajo esta estrategia, claramente convergen productos que ya existen en la región pero que no están generando aún exportaciones competitivas, además se incluyen productos que requieren de capacidades relativamente similares a las existentes en la localidad y que aún no están disponibles para la exportación.

Concretamente en la tabla 2, pueden observarse las oportunidades de diversificación productiva que están "al alcance", en este ranking destacan productos como: Asientos (del sector textil y muebles), estufas y aparatos no eléctricos similares de fundición, hierro o acero, las demás manufacturas de hierro o acero, preparaciones capilares (del sector químico) y remolques y semi remolques. El ranking en extenso puede ser observado a continuación y engloba a los principales 25 productos de la estrategia de selección.

Tabla 2. Ranking de oportunidades: Estrategia al alcance (de corto plazo)

\begin{tabular}{|c|c|c|c|c|c|c|c|}
\hline Sector & Productos específicos de mayor complejidad & $X_{1} * W_{1}$ & $X_{2} * W_{2}$ & $X_{3} * W_{3}$ & $X_{4} * W_{4}$ & ÍNDICE & RANK \\
\hline \multirow{3}{*}{$\begin{array}{l}\text { Textiles y } \\
\text { muebles }\end{array}$} & Asientos** & 1.59 & -0.15 & -0.09 & 0.98 & 2.33 & 1 \\
\hline & Los demás muebles y sus partes** & 1.51 & -0.11 & -0.07 & 0.90 & 2.23 & 4 \\
\hline & Productos textiles acolchados en pieza* & 1.63 & -0.08 & -0.13 & 0.71 & 2.12 & 10 \\
\hline \multirow{6}{*}{ Metales } & $\begin{array}{l}\text { Estufas y aparatos no eléctricos similares de } \\
\text { fundición, hierro o acero** }\end{array}$ & 1.59 & -0.10 & -0.08 & 0.86 & 2.27 & 2 \\
\hline & $\begin{array}{l}\text { Construcciones y sus partes de fundición, hierro o } \\
\text { acero** }\end{array}$ & 1.46 & -0.08 & -0.08 & 0.88 & 2.18 & 6 \\
\hline & Las demás manufacturas de hierro o acero*** & 1.07 & 0.04 & 0.07 & 0.91 & 2.09 & 13 \\
\hline & Telas metálicas de alambre de hierro o acero* & 1.48 & -0.15 & -0.08 & 0.80 & 2.05 & 15 \\
\hline & $\begin{array}{l}\text { Depósitos de fundición, hierro o acero, de } \\
\text { capacidad }<300 \mathrm{l}^{*}\end{array}$ & 1.34 & -0.06 & -0.05 & 0.81 & 2.05 & 16 \\
\hline & Partes de aluminio para construcción* & 1.38 & -0.11 & -0.03 & 0.81 & 2.04 & 18 \\
\hline \multirow{6}{*}{$\begin{array}{l}\text { Químicos } \\
\text { y plásticos }\end{array}$} & Preparaciones capilares* & 1.55 & -0.15 & -0.09 & 0.93 & 2.24 & 3 \\
\hline & Monofilamentos* & 1.63 & -0.11 & -0.06 & 0.71 & 2.17 & 7 \\
\hline & Artículos para la construcción, de plástico** & 1.43 & -0.08 & -0.03 & 0.84 & 2.16 & 8 \\
\hline & Pinturas y barnices, non-acuosos* & 1.32 & -0.08 & -0.01 & 0.81 & 2.03 & 19 \\
\hline & Agentes de limpieza orgánicos (excepto el jabón)* & 1.32 & -0.13 & -0.04 & 0.87 & 2.02 & 21 \\
\hline & Las demás placas de plástico** & 1.19 & -0.03 & 0.00 & 0.85 & 2.01 & 23 \\
\hline \multirow{7}{*}{$\begin{array}{l}\text { Vegetales, } \\
\text { alimentos } \\
\text { y madera }\end{array}$} & Cajones de madera* & 1.62 & -0.12 & -0.05 & 0.76 & 2.20 & 5 \\
\hline & Cerveza de malta* & 1.39 & -0.12 & -0.12 & 0.94 & 2.09 & 11 \\
\hline & Productos de leche, fermentada* & 1.58 & -0.12 & -0.09 & 0.71 & 2.07 & 14 \\
\hline & Chocolates* & 1.32 & -0.11 & -0.06 & 0.87 & 2.03 & 20 \\
\hline & Los demás impresos* & 1.20 & -0.03 & 0.01 & 0.83 & 2.02 & 22 \\
\hline & Otras preparaciones alimenticias* & 1.34 & -0.13 & -0.07 & 0.84 & 1.98 & 24 \\
\hline & Carpintería para edificaciones* & 1.36 & -0.09 & -0.07 & 0.77 & 1.98 & 25 \\
\hline \multirow{3}{*}{ Otros } & Remolques y semirremolques*** & 1.24 & -0.03 & 0.02 & 0.90 & 2.13 & 9 \\
\hline & Refrigeradores, congeladores** & 1.22 & -0.04 & -0.05 & 0.96 & 2.09 & 12 \\
\hline & Vidrieras aislantes de paredes múltiples*** & 1.23 & 0.00 & 0.04 & 0.78 & 2.04 & 17 \\
\hline
\end{tabular}

Nota: Un asterisco $\left(^{*}\right)$ denota una repetición de la oportunidad del producto dentro de las tres estrategias, $\left({ }^{*}\right)$ dos repeticiones y $\left({ }^{* *}\right)$ tres repeticiones. Entre más veces aparezca un determinado producto en los rankings, más factible es que exista un mayor consenso sobre sus virtudes por parte de los actores, públicos y privados, involucrados en el diseño de políticas.

Fuente: Elaboración propia. 
En la estrategia al alcance, el principal reto de los productos con una estrella $\left(^{*}\right)$, es promover su competencia, ya que son bienes que generalmente ya se explotan en la región pero deben mejorar su nivel de competitividad para enriquecer la complejidad económica del estado.

La Estrategia Balanceada (para el mediano plazo): identificó oportunidades en sectores más sofisticados como: maquinaria, vehículos de transporte y otros. Y dejo de enlistar productos de las industrias tradicionales de: "Textiles y muebles", y "vegetales, alimentos y madera".

En la tabla 3, pueden observarse las oportunidades de diversificación productiva que están "balanceadas", es decir, alternativas que otorgan un peso similar a la sofisticación del producto y a su conectividad con otros productos atractivos. En este ranking destacan productos como: Muelles, ballestas y sus hojas, hierro o acero; Artículos textiles para usos técnicos; Vidrieras aislantes de paredes múltiples; Remolques y semirremolques y Partes de vehículos automóviles y tractores. El ranking en extenso puede observarse a continuación:

Tabla 3. Ranking de oportunidades: Estrategia balanceada (de mediano plazo)

\begin{tabular}{|c|c|c|c|c|c|c|c|}
\hline Sector & Productos específicos de mayor complejidad & $X_{1} * W_{1}$ & $X_{2} * W_{2}$ & $X_{3} * W_{3}$ & $X_{4} * W_{4}$ & İNDICE & RANK \\
\hline \multirow{7}{*}{ Metales } & Las demás manufacturas de hierro o acero*** & 0.76 & 0.08 & 0.13 & 0.91 & 1.89 & 1 \\
\hline & Muelles, ballestas y sus hojas, hierro o acero** & 0.43 & 0.19 & 0.29 & 0.87 & 1.78 & 2 \\
\hline & $\begin{array}{l}\text { Radiadores para calefacción central de hierro o } \\
\text { acero** }\end{array}$ & 0.67 & 0.13 & 0.13 & 0.82 & 1.75 & 6 \\
\hline & $\begin{array}{l}\text { Estufas y aparatos no eléctricos similares de } \\
\text { fundición, hierro o acero** }\end{array}$ & 1.13 & -0.20 & -0.16 & 0.86 & 1.64 & 18 \\
\hline & $\begin{array}{l}\text { Guarniciones, herrajes y similares, de metal } \\
\text { común* }\end{array}$ & 0.60 & 0.03 & 0.11 & 0.87 & 1.61 & 22 \\
\hline & $\begin{array}{l}\text { Construcciones y sus partes de fundición, hierro } \\
\text { o acero** }\end{array}$ & 1.04 & -0.16 & -0.16 & 0.88 & 1.61 & 24 \\
\hline & Las demás manufacturas de aluminio** & 0.73 & -0.09 & 0.11 & 0.85 & 1.60 & 25 \\
\hline \multirow{5}{*}{$\begin{array}{l}\text { Químicos y } \\
\text { plásticos }\end{array}$} & Otras manufacturas de caucho vulcanizado** & 0.70 & -0.02 & 0.17 & 0.87 & 1.72 & 9 \\
\hline & Placas de caucho vulcanizado sin endurecer** & 0.60 & 0.12 & 0.23 & 0.73 & 1.68 & 11 \\
\hline & Las demás placas de plástico** & 0.85 & -0.05 & 0.00 & 0.85 & 1.64 & 16 \\
\hline & Artículos para la construcción, de plástico** & 1.02 & -0.16 & -0.06 & 0.84 & 1.64 & 17 \\
\hline & Masilla* & 0.78 & -0.03 & 0.11 & 0.75 & 1.61 & 23 \\
\hline \multirow{3}{*}{$\begin{array}{c}\text { Textiles y } \\
\text { muebles }\end{array}$} & Artículos textiles para usos técnicos** & 0.53 & 0.24 & 0.18 & 0.80 & 1.76 & 5 \\
\hline & Asientos $^{* *}$ & 1.13 & -0.30 & -0.18 & 0.98 & 1.63 & 19 \\
\hline & Los demás muebles y sus partes** & 1.08 & -0.22 & -0.13 & 0.90 & 1.62 & 21 \\
\hline \multirow{5}{*}{ Maquinaria } & Bombas, compresores, ventiladores, etc. ${ }^{* *}$ & 0.25 & 0.22 & 0.33 & 0.93 & 1.75 & 7 \\
\hline & Artículos de grifería para tuberías, calderas, etc. ${ }^{* *}$ & 0.13 & 0.25 & 0.39 & 0.93 & 1.70 & 10 \\
\hline & Refrigeradores, congeladores** & 0.87 & -0.08 & -0.10 & 0.96 & 1.65 & 13 \\
\hline & Partes para motores de encendido por chispa** & 0.36 & 0.08 & 0.25 & 0.96 & 1.64 & 15 \\
\hline & Arboles de transmisión ${ }^{* *}$ & 0.00 & 0.37 & 0.37 & 0.89 & 1.63 & 20 \\
\hline \multirow{2}{*}{$\begin{array}{l}\text { Vehículos de } \\
\text { transporte }\end{array}$} & Remolques y semirremolques*** & 0.88 & -0.06 & 0.05 & 0.90 & 1.77 & 3 \\
\hline & Partes de vehículos automóviles y tractores** & 0.28 & 0.14 & 0.32 & 1.03 & 1.77 & 4 \\
\hline \multirow{3}{*}{ Otros } & Señales eléctricas para vías** & 0.54 & 0.13 & 0.18 & 0.80 & 1.65 & 14 \\
\hline & Vidrieras aislantes de paredes múltiples*** & 0.88 & 0.00 & 0.08 & 0.78 & 1.73 & 8 \\
\hline & Los demás impresos** & 0.86 & -0.05 & 0.02 & 0.83 & 1.66 & 12 \\
\hline
\end{tabular}

Nota: Un asterisco $\left.{ }^{*}\right)$ denota una repetición de la oportunidad del producto dentro de las tres estrategias, $\left({ }^{* *}\right)$ dos repeticiones y $\left({ }^{* * *}\right)$ tres repeticiones. Entre más veces aparezca un

determinado producto en los rankings, más factible es que exista un mayor consenso sobre sus virtudes por parte de los actores, públicos y privados, involucrados en el diseño de políticas.

Fuente: Elaboración propia.

Finalmente, la Estrategia de Apuestas a futuro (para el largo plazo): identificó oportunidades en sectores de alto potencial para la diversificación como: "electrónicos" "piedra y vidrio". Las principales oportunidades dentro de este ranking, se encuentran conglomeradas en los sectores de "Maquinaria" y "Metales". No obstante, el sector automotriz, tiene en primer lugar la oportunidad estratégica de producir "partes de vehículos automóviles y tractores". 
REMEF (The Mexican Journal of Economics and Finance) Diagnóstico de la complejidad económica del estado de Hidalgo: de las capacidades a las oportunidades

Tabla 4. Ranking de oportunidades: Apuesta estratégica (de largo plazo)

\begin{tabular}{|c|c|c|c|c|c|c|c|}
\hline Sector & Productos específicos de mayor complejidad & $X_{1} * W_{1}$ & $X_{2} * W_{2}$ & $X_{3} * W_{3}$ & $X_{4} * W_{4}$ & INDICE & RANK \\
\hline \multirow{2}{*}{$\begin{array}{l}\text { Vehículos de } \\
\text { transporte }\end{array}$} & $\begin{array}{l}\text { Partes de vehículos automóviles y } \\
\text { tractores** }\end{array}$ & 0.22 & 0.07 & 0.63 & 1.03 & 1.96 & 1 \\
\hline & Remolques y semirremolques*** & 0.71 & -0.03 & 0.09 & 0.90 & 1.67 & 19 \\
\hline \multirow{4}{*}{ Metales } & $\begin{array}{l}\text { Muelles, ballestas y sus hojas, hierro o } \\
\text { acero** }\end{array}$ & 0.35 & 0.10 & 0.57 & 0.87 & 1.89 & 4 \\
\hline & $\begin{array}{l}\text { Las demás manufacturas de hierro o } \\
\text { acero*** }\end{array}$ & 0.61 & 0.04 & 0.26 & 0.91 & 1.83 & 5 \\
\hline & $\begin{array}{l}\text { Radiadores para calefacción central de hierro o } \\
\text { acero** }\end{array}$ & 0.53 & 0.07 & 0.25 & 0.82 & 1.67 & 18 \\
\hline & Las demás manufacturas de aluminio** & 0.59 & -0.04 & 0.22 & 0.85 & 1.61 & 25 \\
\hline \multirow{3}{*}{$\begin{array}{l}\text { Químicos y } \\
\text { plásticos }\end{array}$} & Otras manufacturas de caucho vulcanizado** & 0.56 & -0.01 & 0.34 & 0.87 & 1.76 & 9 \\
\hline & Placas de caucho vulcanizado sin endurecer** & 0.48 & 0.06 & 0.45 & 0.73 & 1.73 & 12 \\
\hline & Tubos de caucho vulcanizado sin endurecer* & 0.25 & 0.04 & 0.53 & 0.86 & 1.69 & 15 \\
\hline \multirow{10}{*}{ Maquinaria } & $\begin{array}{l}\text { Artículos de grifería para tuberías, calderas, } \\
\text { etc.** }\end{array}$ & 0.10 & 0.12 & 0.79 & 0.93 & 1.95 & 2 \\
\hline & Bombas, compresores, ventiladores, etc.** & 0.20 & 0.11 & 0.67 & 0.93 & 1.92 & 3 \\
\hline & Arboles de transmisión** & 0.00 & 0.18 & 0.74 & 0.89 & 1.82 & 6 \\
\hline & Partes para motores de encendido por chispa** & 0.29 & 0.04 & 0.50 & 0.96 & 1.78 & 7 \\
\hline & $\begin{array}{l}\text { Motores de embolo de encendido por } \\
\text { compresión* }\end{array}$ & -0.04 & 0.13 & 0.76 & 0.93 & 1.77 & 8 \\
\hline & Bombas para líquidos* & 0.04 & 0.10 & 0.69 & 0.93 & 1.75 & 10 \\
\hline & Las demás máquinas de elevación* & 0.18 & 0.09 & 0.63 & 0.84 & 1.73 & 11 \\
\hline & $\begin{array}{l}\text { Aparatos para tratar materias mediante cambio } \\
\text { de temperatura* }\end{array}$ & -0.04 & 0.07 & 0.79 & 0.89 & 1.72 & 13 \\
\hline & Aparatos para regulación automáticos* & 0.20 & 0.06 & 0.49 & 0.93 & 1.68 & 17 \\
\hline & Centrifugadoras* & 0.20 & 0.08 & 0.45 & 0.93 & 1.65 & 22 \\
\hline \multirow{3}{*}{ Electrónicos } & $\begin{array}{l}\text { Partes de aparatos relacionados con circuitos } \\
\text { eléctricos* }\end{array}$ & 0.37 & 0.01 & 0.38 & 0.91 & 1.67 & 20 \\
\hline & Aparatos de alumbrado para automóviles* & 0.27 & 0.02 & 0.45 & 0.92 & 1.66 & 21 \\
\hline & Señales eléctricas para vías* & 0.43 & 0.07 & 0.36 & 0.80 & 1.65 & 23 \\
\hline \multirow{2}{*}{ Piedra y vidrio } & Abrasivos naturales o artificiales en polvo* & 0.03 & 0.10 & 0.73 & 0.81 & 1.68 & 16 \\
\hline & Vidrieras aislantes de paredes múltiples*** & 0.70 & 0.00 & 0.15 & 0.78 & 1.63 & 24 \\
\hline Otro & Artículos textiles para usos técnicos** & 0.43 & 0.12 & 0.36 & 0.80 & 1.71 & 14 \\
\hline
\end{tabular}

Nota: Un asterisco $\left(^{*}\right)$ denota una repetición de la oportunidad del producto dentro de las tres estrategias, $(* *)$ dos repeticiones y $(* * *)$ tres repeticiones. Entre más veces aparezca un

determinado producto en los rankings, más factible es que exista un mayor consenso sobre sus virtudes por parte de los actores, públicos y privados, involucrados en el diseño de políticas.

Fuente: Elaboración propia.

\section{Interpretaciones extendidas de los resultados}

Una mejor interpretación de los resultados, se logra ligando los hallazgos del mapeo ubicuidad y diversidad con los rankings de oportunidades detectados. De esta manera, se evidencia que para el estado de Hidalgo, es prioritario poner énfasis en la estrategia balanceada, debido a que sí en materia de ubicuidad-diversidad, la entidad se encuentra claramente en el cuadrante que engloba mayor diversificación promedio con mayor ubicuidad promedio. Es necesario por consecuencia identificar oportunidades que den similar importancia a la sofisticación del producto (menor ubicuidad) y a su conectividad con otros productos atractivos (diversidad). Por ende, se asume que la estrategia al alcance sería relativamente limitada dados los retos actuales de la entidad mientras que la apuesta estratégica, podría contemplarse como prospectiva para el largo plazo. 
Tabla 5. Sectores de alto potencial para la diversificación del estado de Hidalgo hacia productos de mayor complejidad: estrategia balanceada.

\begin{tabular}{|c|c|c|c|}
\hline Sectores & Productos específicos de mayor complejidad & $\begin{array}{c}\text { Polos de } \\
\text { vocacionamiento }\end{array}$ & $\begin{array}{l}\text { Polo de generación de } \\
\text { nuevas capacidades }\end{array}$ \\
\hline \multirow{7}{*}{ Metales } & Las demás manufacturas de hierro o acero & \multirow{7}{*}{$\begin{array}{l}\text { Región Huichapan, } \\
\text { Corredor Industrial } \\
\text { del Altiplano, } \\
\text { Valle de Tizayuca }\end{array}$} & \multirow{22}{*}{$\begin{array}{l}\text { Pachuca: Ciudad del } \\
\text { conocimiento y la cultura }\end{array}$} \\
\hline & Muelles, ballestas y sus hojas, hierro o acero & & \\
\hline & Radiadores para calefacción central de hierro o acero & & \\
\hline & $\begin{array}{l}\text { Estufas y aparatos no eléctricos similares de fundición, } \\
\text { hierro o acero }\end{array}$ & & \\
\hline & Guarniciones, herrajes y similares, de metal común & & \\
\hline & $\begin{array}{l}\text { Construcciones y sus partes de fundición, hierro } \\
\text { o acero }\end{array}$ & & \\
\hline & Las demás manufacturas de aluminio & & \\
\hline \multirow{5}{*}{$\begin{array}{l}\text { Químicos y } \\
\text { plásticos }\end{array}$} & Otras manufacturas de caucho vulcanizado & \multirow{5}{*}{$\begin{array}{l}\text { Región Huichapan, } \\
\text { Valle de Tulancingo }\end{array}$} & \\
\hline & Placas de caucho vulcanizado sin endurecer & & \\
\hline & Las demás placas de plástico & & \\
\hline & Artículos para la construcción, de plástico & & \\
\hline & Masilla & & \\
\hline \multirow{3}{*}{$\begin{array}{l}\text { Textiles y } \\
\text { muebles }\end{array}$} & Artículos textiles para usos técnicos & \multirow{3}{*}{ Valle de Tulancingo } & \\
\hline & Asientos & & \\
\hline & Los demás muebles y sus partes & & \\
\hline \multirow{5}{*}{ Maquinaria } & Bombas, compresores, ventiladores, etc. & \multirow{5}{*}{$\begin{array}{l}\text { Valle de Tizayuca, } \\
\text { Región Tula Tepeji }\end{array}$} & \\
\hline & Artículos de grifería para tuberías, calderas, etc. & & \\
\hline & Refrigeradores, congeladores & & \\
\hline & Partes para motores de encendido por chispa & & \\
\hline & Arboles de transmisión & & \\
\hline \multirow{2}{*}{$\begin{array}{l}\text { Vehículos de } \\
\text { transporte }\end{array}$} & Remolques y semirremolques & \multirow{2}{*}{$\begin{array}{l}\text { Corredor Industrial del } \\
\text { Altiplano, Valle de Tizayuca }\end{array}$} & \\
\hline & Partes de vehículos automóviles y tractores & & \\
\hline
\end{tabular}

Fuente: Elaboración propia con base a resultados y CITNOVA (2016).

Figura 2. Hidalgo: polos de desarrollo y vocacionamiento productivo

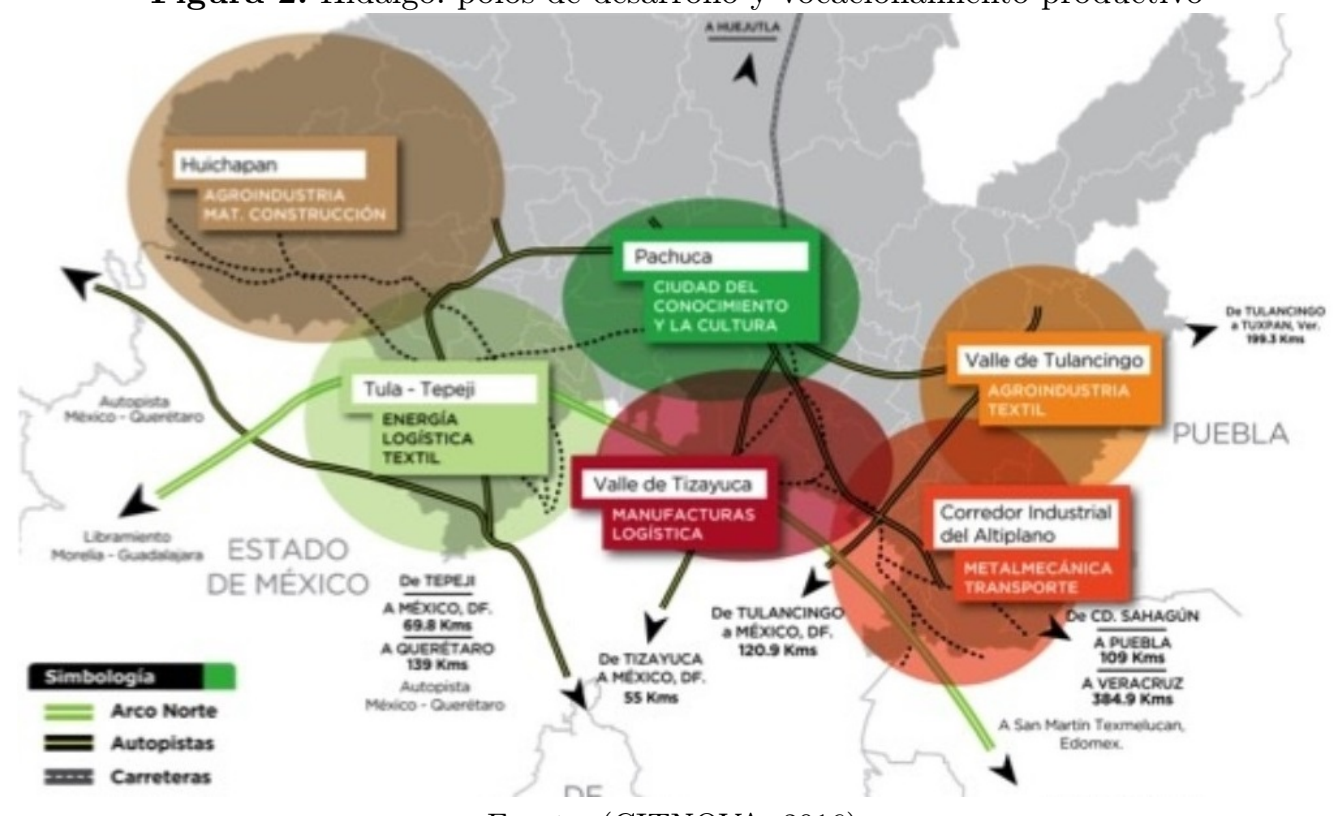

Fuente: (CITNOVA, 2016).

Los tres rankings de oportunidades generados, muestran la existencia de un gran potencial para que diferentes regiones hidalguenses se muevan de manera gradual hacia productos e industrias de mayor sofisticación basados pues en sus propias capacidades de partida y proyectadas. No obstante, estos movimientos graduales, se lograrán alcanzar y consolidar con tres ingredientes esenciales: la intensión emprendedora, los incentivos gubernamentales que la apoyen y la creación de nuevas capacidades. 
REMEF (The Mexican Journal of Economics and Finance) Diagnóstico de la complejidad económica del estado de Hidalgo:

Con lo que respecta a la intensión emprendedora, estos rankings pretenden ser una brújula de emprendimiento cuya finalidad no es limitar la voluntad y creatividad emprendedora sino más bien, orientar la vista de los empresarios, emprendedores e inversionistas hacia claros sectores y productos que auguran mayor complejidad y sofisticación factiblemente alcanzable dadas las capacidades actuales-potenciales a nivel local. Claramente, las oportunidades productivas (aquí detectadas) deben traslapar con las oportunidades de mercado identificadas por los emprendedores. Por ende, tal como advierte Rodríguez Jiménez (2005, p.87), "la comprensión de la situación empresarial necesita un examen profundo de la relación entre emprendedor, la naturaleza de su proyecto y su inserción en las redes relacionales", por lo que este tipo rankings puede orientar al emprendedor y a sus redes a la validación de la oportunidad de emprendimiento. Ya que tal como indica Castañeda (2018), para que exista una discusión informada entre todos los actores empresariales es imprescindible aplicar metodologías rigurosas que contribuyan a identificar nuevas industrias que pudieran ser deseables para la sociedad desde distintos puntos de vista, porque al final "el descubrimiento de oportunidades es una función de la distribución de la información en la sociedad" (Shane y Venkataraman, 2000).

Por otro lado, Huerta (2016), refiere que en el estado de Hidalgo, es necesario que exista una empatía plena entre las vocaciones económicas y una política educativa acorde con las necesidades reales. En otras palabras, es prioritario ir desarrollando capacidades y recursos humanos acordes con las oportunidades productivas de diversificación detectadas a lo largo y ancho del territorio hidalguense, promoviendo la movilidad de celebros, pero evitando la fuga de talentos. Ya que "la movilidad de capacidades a escala nacional y regional es importante porque resulta más rápido y barato mover un cerebro que crearlo" (Hausmann, 2017). Pero, resulta aún más óptimo aprovechar un cerebro local y atraer las capacidades locales que beneficien la economía y la generación de empleos en la región. El sector gubernamental y el sector académico, deberán velar por generar un círculo virtuoso en donde la demanda de recursos humanos especializados (reflejados en la estrategia de largo plazo o apuesta estratégica de oportunidades) empate con la oferta académica generada y proyectada.

Lo anterior es relevante si se reconoce que "la complejidad económica no se genera por sí sola dado que la falta de capacidades puede impedir a las localidades acceder a industrias más sofisticadas y conectadas en la red mundial de productos transables" (Castañeda, 2018, p.52).

Adicionalmente, según el Periódico Oficial del Estado de Hidalgo (2017), el Consejo Rector de Pachuca Ciudad del Conocimiento y la Cultura, tiene entre sus atribuciones la encomienda de crear nuevas oportunidades de negocio y agregar valor a las empresas maduras alojadas en la región. Por consecuencia, la identificación de oportunidades antecede a la creación de las mismas, y es por ello que se debe tomar en cuenta los rankings de oportunidades, ya que éstos, constituyen un primer intento por orientar los esfuerzos de emprendimiento y diversificación hacia sectores y productos cuyas capacidades subyacentes necesarias para elaborarlos se encuentran presentes y/o latentes dentro de la región estudiada. Por lo tanto, es posible evidenciar que incluso las empresas provenientes de sectores maduros, pueden generar productos que les permitan elevar su sofisticación exportadora y emprendedora. Paralelamente, sectores emergentes pueden ser impulsados a sabiendas que éstos tienen ya directa o indirectamente una plataforma de capacidades que puede impulsar su éxito. 


\section{Conclusiones y reflexiones}

La presente investigación surgió para responder por vez primera la interrogante de: ¿Cuáles son las oportunidades concretas de diversificación productiva factibles en el corto, mediano y largo plazo identificadas a partir de las capacidades productivas existentes en el estado de Hidalgo? Por ende, el objetivo central consistió en identificar un ranking de oportunidades de emprendimiento y diversificación productiva que resulta útil para diversos agentes.

Para tal propósito, se optó por adoptar el método cuantitativo, haciendo uso del Método de Reflexiones (MR) y la asignación de ponderadores, con base en las variables de la complejidad económica propuestas por Hidalgo Hausmann (2009) y recopilando los datos procedentes del Atlas de complejidad (2018). A partir de las operaciones algebraicas, se halla en un primer momento, que el estado de Hidalgo se encuentra claramente ubicado en el cuadrante que refleja: "mayor diversificación promedio con mayor ubicuidad promedio". En un segundo momento, la estrategia balanceada de selección de oportunidades logra identificar concretamente cuales son los sectores industriales que ofrecen las mejores posibilidades de diversificación productiva para incrementar la complejidad económica, principalmente: Metales, Químicos y plásticos, Textiles y muebles, Maquinaria y Vehículos de transporte.

En ese sentido, los hallazgos revelan también que los principales candidatos (oportunidades) para movilizar esta transformación hacia una mayor complejidad son los productos de: Las demás manufacturas de hierro o acero; Muelles, ballestas y sus hojas, hierro o acero; Radiadores para calefacción central de hierro o acero; Estufas y aparatos no eléctricos similares de fundición, hierro o acero; Guarniciones, herrajes y similares, de metal común; Construcciones y sus partes de fundición, hierro o acero; Las demás manufacturas de aluminio; Otras manufacturas de caucho vulcanizado; Placas de caucho vulcanizado sin endurecer; Las demás placas de plástico; Artículos para la construcción, de plástico; Masilla; Artículos textiles para usos técnicos; Asientos; Los demás muebles y sus partes; Bombas, compresores, ventiladores, etc; Artículos de grifería para tuberías, calderas, etc; Refrigeradores, congeladores; Partes para motores de encendido por chispa; Arboles de transmisión; Remolques y semirremolques; Partes de vehículos automóviles y tractores.

Adicionalmente, los hallazgos del presente trabajo ofrecen un consenso con el Plan Estatal de Desarrollo (2016-2022), y la Agenda Estatal de Innovación (2015) en cuanto a la consideración de la industria "Metalmecánica/transporte/automotriz" como sector primordialmente estratégico. Esto implica que la región hidalguense ostenta capacidades reales y potenciales para impulsar estos sectores y generar oportunidades de emprendimiento y diversificación productiva que impacte en la complejidad económica de la región.

Se refuerza entonces la premisa de que "la perspectiva de la capacidad puede ser muy importante para juzgar las oportunidades. Dado que la capacidad es, en efecto, nada más que una perspectiva desde el punto de vista de la cual se pueden evaluar en forma más razonable las ventajas y desventajas" (Sen, 2015, p.326).

Cabe señalar que los rankings de oportunidades generados no son prescriptivos en ningún momento. Por lo que tal como sugieren Hausmann, Cheston Santos (2015, p.28) "deben ser interpretados como una aproximación a la identificación de oportunidades de diversificación productiva, y no como un proceso a través del cual se seleccionan sectores ganadores. Esta definición debe ser la base de un proceso iterativo de análisis que valide efectivamente su pertinencia, y provea una hoja de ruta para identificar posibles barreras que podrian estar inhibiendo su surgimiento, tales como capacidades especificas ausentes, bienes públicos u otras fallas de mercado".

En ese sentido, es importante recordar que los cálculos de ubicuidad y diversidad fueron hechos sobre las actividades netamente industriales. Por lo que el sector terciario sale de la lupa de nuestro análisis junto con parte del sector perteneciente a la economía 
REMEF (The Mexican Journal of Economics and Finance) Diagnóstico de la complejidad económica del estado de Hidalgo:

naranja $a^{5}$, del mismo modo, los datos de complejidad económica están basados en la economía formal, por lo que claramente existe una infravaloración de la actividad económica efectivamente presente. No obstante las limitaciones anteriores, es indiscutible que los rankings de oportunidades resultan funcionales porque se acogen a la premisa que "apalancándose en las capacidades que ya existen, las entidades puede ser capaces de moverse paulatinamente hacia actividades de mayor complejidad" (Hausmann, Cheston Santos, 2015).

Adicionalmente, se considera necesario ampliar los estudios de complejidad económica hacia aquellos estados en los que actualmente se carece de evidencia empírica que revele sus propios rankings de oportunidades. Tal es el caso de los estados de: Baja California Sur, Ciudad de México, Coahuila, Colima, Durango, Jalisco, Morelos, Nayarit, Quintana Roo, Sinaloa, Sonora, Tamaulipas, Tlaxcala y Zacatecas.

Finalmente se concluye que futuras líneas de investigación deberían enfocarse al estudio del proceso de acumulación de capacidades, propiamente a las capacidades de absorción para entonces tener un mayor entendimiento sobre cómo y dónde se genera la capacidad de aprender y generar nuevos productos.

\section{Referencias}

Abdon, A. Felipe, J. (2010), "Product space: ¿qué nos dice sobre las oportunidades de crecimiento y transformación estructural de áfrica subsahariana?" Fundación Ideas

Ackoff, R. (2000), Cápsulas de Ackoff: Administración en pequeñas dosis. México: LIMUSA.

Agendas de Innovación (2015), "Agenda de Innovación de Hidalgo". CONACYT. Disponible en: http: $/ /$ www . agendasinnovacion org $/ ? \mathrm{p}=1350$

Atlas de complejidad. (2018), "Atlas Mexicano de Complejidad Económica". Disponible en: http:// complejidad.datos.gob.mx/\#/downloads, [Recuperado 10 marzo 2017].

BID (2015), "Economía Naranja: Innovaciones que no sabias que eran de América Latina y el Caribe". Banco Interamericano de Desarrollo.

Castañeda, G. (2018), "Reportes Subnacionales de Complejidad Económica". Disponible en: https:// www.gob.mx/productividad/documentos/reportes-subnacionales-de-complejidad-economica

Cepal. (2005), "Indicadores sociales en América Latina y el Caribe". Disponible en: http://www. cepal. org/es/publicaciones/4735-indicadores-sociales-en-america-latina-y-el-caribe

CITNOVA, (2016), "Ciudades que crean y atraen talento innovador y emprendedor: programas y proyectos". Disponible en: http://fororedemprendia.blogspot.mx/2015/10/ciudades-del-conocimiento-foro_ 20. html?m=1

Davidsson, P. (2015), "Entrepreneurial opportunities and the entrepreneurship nexus: A re-conceptualization". Journal of Business Venturing, http://dx.doi.org/10.1016/j.jbusvent.2015.01.002.

Felipe, J., Kumar, u., Abdon, A. (2010), "As You Sow So Shall You Reap: From Capabilities to Opportunities". Working Paper No. 613. Levi Economic Institute.

Felipe, J., Kumar, U., Abdon, A., Bacate, M. (2012), "Product complexity and economic development". Structural Change and Economic Dynamics, 23(1), 36-68. doi:10.1016/j.strueco.2011.

GEM. (2005), "Reporte Nacional de Chile". Global Entrepreneurship Monitor

González-Álvarez, N., Solís-Rodríguez, V. (2011), "Descubrimiento de oportunidades empresariales: capital humano, capital social y género". INNOVAR. Revista de Ciencias Administrativas y Sociales, 21 (41), 187-196.

Hartmann, M., et al (2017), "Linking Economic Complexity, Institutions, and Income World Development, Inequality" 93(2017) pp. 75-93, ISSN 0305-750X, https://doi.org/10.1016/j.worlddev.2016.12.020.

Hausmann, R., Hidalgo, C. A. (2013), "How will the Netherlands earn its income 20 years from now? A growth ventures analysis for the Netherlands". The Hague: The Netherlands Scientic Council for Government Policy (WRR). Disponible en: http://www.wrr.nl/en/publications/publication/ article/how-will-thenetherlands-ea!rn-its-income-20-years-from-now/

Hausmann, R., Hidalgo, C; et al. (2011), "The Atlas of Economic Complexity: Mapping paths to prosperity". MIT Harvard University. Disponible en: http://atlas.cid.harvard.edu/media/atlas/pdf/ HarvardMIT_AtlasOfEconomicComplexity_Part_I.pdf

\footnotetext{
${ }^{5}$ Arte y patrimonio: Artes visuales, artes escénicas y espectáculos, turismo y patrimonio cultural material e inmaterial. Industrias culturales convencionales: Editorial, audiovisual, fonográfica. Creaciones funcionales, nuevos medios y software: diseño, software de contenidos como videojuegos (BID, 2015).
} 
Haussmann, R., Cheston, T., Santos, M.A. (2015), "La complejidad económica de Chiapas: análisis de capacidades y posibilidades de diversificación productiva". Center for International Development at Harvard University. Disponible en: http://growthlab.cid.harvard.edu/files/growthlab/files/ cid_wp_302.pdf

Hausmann, R., Hidalgo, C., Bustos, S., Coscia, M., Chung, S., Jimenez, J., Yıldırım, M. (2014), "The atlas of economic complexity - Mapping paths to prosperity". Cambridge, MA: MIT Press.

Hidalgo, C. A., Hausmann, R. (2009), "The building blocks of economic complexity". Proceedings of the National Academy of Sciences, USA, 106(26), 10570-5. doi:10.1073/pnas.0900943106

Hidalgo, C. (2017), "Why information grows: The Evolution of Order, from Atoms to Economies". Basic Books

Huerta, A. (2016), Entrevista al director del CITNOVA. (Carla Pérez, Entrevistador)

Nepelski, D., De Prato, G. (2015), "Corporate control, location and complexity of ICT RD: A network analysis at the city level". Urban Studies, 52(4), 721-737.

Oppenheimer. (2014), "Crear o morir: la esperanza de América latina y las 5 claves de la innovación". México: DEBATE

Periódico Oficial del Estado de Hidalgo (2017), "Consejo Rector de Pachuca Ciudad del Conocimiento y la Cultura". Disponible: http://transparencia.hidalgo.gob.mx/descargables/ENTIDADES/ CCConocimiento/2facultades.pdf

Pinheiro, F. et al, (2018), "Shooting High or Low: Do Countries Benefit from Entering Unrelated Activities?" Cornell University

Plan Estatal de Desarrollo (2016), "El Plan Estatal de Desarrollo 2016-2022”. Disponible en el Observatorio Tecnológico de Hidalgo: http://otech.uaeh.edu.mx/estado-de-hidalgo.html

Rodríguez, C., Jiménez, M. (2005), "Emprenderismo, acción gubernamental y academia". Revisión de la literatura. INNOVAR. Revista de Ciencias Administrativas y Sociales, 15 (26), 73-89.

Sen A. (2015), "La idea de la justicia". México: Taurus.

Shane, S., Venkataraman, S. (2000), "The promise of entrepreneurship as a field of research". Academy of Management Review, 25(1), 217-226.

Shane, S. (2000), "Prior knowledge and the discovery of entrepreneurial opportunities". Organization Science, 11(4), 448-469.

Simoes, A., Hidalgo, C. (2011), "The economic complexity observatory: An analytical tool for understanding the dynamics of economic development". Fifth AAAI Conference on Artificial Intelligence Works. 